\title{
Signal and Noise Characteristics of Patterned Media
}

\author{
Mustafa M. Aziz, Member, IEEE, C. David Wright, Member, IEEE, Barry K. Middleton, Member, IEEE, Huan Du, \\ and Paul Nutter
}

\begin{abstract}
Analytical expressions of the replay spectra from single- and multiple-recorded bits in patterned media have been derived. The expressions were extended to include position jitter noise and bit-size variations.
\end{abstract}

Index Terms-Noise, patterned media, signal.

\section{INTRODUCTION}

$\mathbf{T}$ HE AIM of this paper is to provide simple analytical expressions of the replay spectra from isolated and single rows of rectangular magnetic islands in recorded patterned media. Furthermore, noise arising from bit-position jitter and bit-size variations is also modeled.

The theoretical development of signal and noise in this paper follows the methods used in magnetic recording theory on continuous media [1]. There are, however, differences between the two systems since the replay transducers in patterned media are expected to have dimensions comparable to the magnetic bits [2]. In addition, the nonmagnetic spacings between the magnetic bits will introduce additional nulls in replay spectra at wavelengths comparable to the bit length.

\section{HEAD FIELDS}

The generality of the spectral theory presented in this paper allows field functions for any transducer type or shape to be used. This includes inductive, magneto-resistive [3] and magnetic force type transducers [4]. In the following analysis, the single pole head will be utilized as the replay transducer assuming that the track width is greater than the bit width. For simplicity, the far-field approximations to the head field are used here with components defined as [1]

$$
\begin{aligned}
& H_{x}^{p}(x, y)=\frac{C I \eta}{\pi} \cdot \frac{x}{\left(x^{2}+y^{2}\right)} \\
& H_{y}^{p}(x, y)=\frac{C I \eta}{\pi} \cdot \frac{y}{\left(x^{2}+y^{2}\right)}
\end{aligned}
$$

where $I$ is the current in the replay head of $C$ coil turns and efficiency $\eta$. As indicated in Fig. 1(a), $g$ is the pole length and $y$ is the distance from the head surface. The Fourier transforms of the $x$ and $y$ components of the head field are given, respectively,

\footnotetext{
Manuscript received February 15, 2002; revised May 20, 2002. This work was supported by the Engineering and Physical Sciences Research Council under Grants GR/M46648 and GR/L83462.

M. M. Aziz, C. D. Wright, and H. Du are with the School of Engineering and Computer Science, University of Exeter, Exeter EX4 4QF, U.K. (e-mail: M.M.Aziz@exeter.ac.uk; David.Wright@exeter.ac.uk; H.Du@exeter.ac.uk).

B. K. Middleton and P. Nutter is with the Department of Computer Science, University of Manchester, Oxford Road, Manchester M13 9PL, UK. Tel.: +44 (0)161 2754551 (e-mail: B.K.Middleton@cs.man.ac.uk).

Digital Object Identifier 10.1109/TMAG.2002.802787.
}

by $H_{x}^{p}(k, y)=j C I \eta \cdot e^{-k y}$ and $H_{y}^{p}(k, y)=C I \eta \cdot e^{-k y}$, where $k=2 \pi / \lambda$ is the wavenumber and $\lambda$ is the wavelength.

\section{SIGNAL}

\section{A. Single Bits}

Individual magnetic bits are assumed to have a rectangular geometry with length $l$, thickness $\delta$, and width $w$ as indicated in Fig. 1(a). The replay flux in the head coil is found from reciprocity [5]

$$
\Phi(\bar{x})=\mu_{o} \int_{z} \int_{y} \int_{x} M(x, y, z) \cdot \boldsymbol{H}^{p}(x+\bar{x}, y, z) d x d y d z
$$

where $\mu_{o}$ is the permeability of free space. In two dimensions, the head field $\boldsymbol{H}^{p}$ is given by (1), and the magnetization inside each individual bit is given by $\boldsymbol{M}=M \cos \theta \hat{x}+M \sin \theta \hat{y}$ with magnitude $M$ and orientation $\theta$, as shown in Fig. 1(b). $\hat{x}$ and $\hat{y}$ are unit vectors in the $x$ and $y$ directions, respectively. Thus, (2) reduces to

$$
\begin{aligned}
\Phi(\bar{x})=\alpha \mu_{o} M \int_{z=-w / 2}^{w / 2} & \int_{y=d}^{d+\delta} \int_{x=-l / 2}^{l / 2}\left\{\cos \theta \cdot H_{x}^{p}(x+\bar{x}, y)\right. \\
& \left.+\sin \theta \cdot H_{y}^{p}(x+\bar{x}, y)\right\} d x d y d z
\end{aligned}
$$

where $\alpha= \pm 1$ to indicate the direction of magnetization. Taking the Fourier transform of (3) with $\bar{x}$ as the operator yields

$$
\begin{aligned}
\Phi(k)=\alpha \mu_{o} M \int_{x=-w / 2}^{w / 2} & \int_{y=d}^{d+\delta} \int_{x=-l / 2}^{l / 2}\left\{\cos \theta \cdot H_{x}^{p}(k, y) e^{j k x}\right. \\
& \left.+\sin \theta \cdot H_{y}^{p}(k, y) e^{j k x}\right\} d x d y d z
\end{aligned}
$$

In the absence of a soft underlayer, integration over the volume of the magnetic bit yields the replay flux as

$$
\begin{aligned}
\Phi(k)=\alpha \mu_{o} M w l \delta \cdot(\cos \theta-j \operatorname{sgn}(k) \sin \theta) \\
\cdot\left[H_{s}(k)\right] \cdot\left[\frac{\sin \left(\frac{k l}{2}\right)}{\left(\frac{k l}{2}\right)}\right] \cdot\left[e^{-k d}\right] \cdot\left[\frac{1-e^{-k \delta}}{k \delta}\right]
\end{aligned}
$$

where $H_{s}(k)$ is the Fourier transform of the head-field function at the head surface $(y=0)$. The term $\sin (k l / 2) /(k l / 2)$ in (5) indicates a null in the replay spectrum at a wavelength equal to the bit length $l$. The replay voltage is given by the spatial derivative of (3) with respect to $\bar{x}$ and, hence, in the Fourier domain is simply

$$
V(k)=j k v \Phi(k)
$$

where $v$ is the head-to-medium speed. 


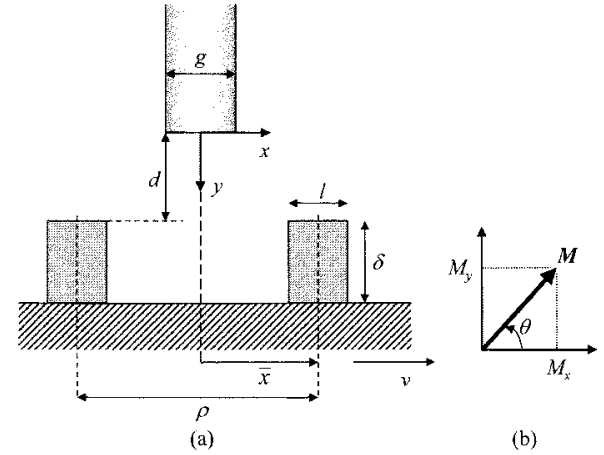

Fig. 1. (a) Coordinate system and magnetic bit size. (b) Magnetization vector of individual magnetic bits.

\section{B. Multiple Bits}

For a single infinite row of recorded islands magnetized in the same direction and separated by a distance $\rho$ from their centers as shown in Fig. 1(a), the replay voltage can be written as the superposition of the voltages from isolated magnetic islands in the form

$$
V_{s}(\bar{x})=\sum_{n=-\infty}^{\infty} V(\bar{x}-n \rho)
$$

Taking the Fourier transform of (7) yields

$$
V_{s}(k)=k_{o} V(k) \sum_{n=-\infty}^{\infty} \delta\left(k-n k_{o}\right)
$$

where $k_{o}=2 \pi / \rho$. Similarly for an alternating sequence of magnetized bits (with wavelength $2 \rho$ ), the Fourier transform of the replay voltage was found to be

$$
V_{s}(k)=k_{o} V(k) \sum_{n=-\infty}^{\infty} \delta\left(k-(2 n+1) k_{o}\right)
$$

\section{NOISE}

Noise in patterned media is modeled as jitter in the bit positions and variations in the bit sizes. Including position jitter, $s$, the replay signal for an "all ones" sequence can be written as

$$
V_{s}(\bar{x})=\sum_{n=-\infty}^{\infty} V\left(\bar{x}-n \rho-s_{n}\right)
$$

which for small perturbations in $s_{n}$, can be expanded to a firstorder Taylor series

$$
V_{s}(\bar{x})=\sum_{n=-\infty}^{\infty} V(\bar{x}-n \rho)+\sum_{n=-\infty}^{\infty} s_{n} \frac{\partial V(\bar{x}-n \rho)}{\partial \bar{x}}
$$

The spatial autocorrelation function of (10) is given by

$$
R(\tau)=\lim _{L \rightarrow \infty} \frac{1}{L} \int_{\bar{x}=-L / 2}^{L / 2} V_{s}(\bar{x}) V_{s}(\bar{x}-\tau) d \bar{x}
$$

where $L=N \rho$ is the averaging length with $N$ periods. Evaluating the autocorrelation function assuming that there are no noise correlations between neighboring bits and that $s_{n}$ is a zero-mean random variable yields

$$
\begin{array}{r}
R(\tau)=\lim _{N \rightarrow \infty} \frac{1}{N \rho} \sum_{n=-N}^{N} \int_{-N \rho / 2}^{N \rho / 2}\{V(\bar{x}-n \rho) V(\bar{x}-n \rho-\tau) \\
\left.+s_{n}^{2} \frac{\partial V(\bar{x}-n \rho)}{\partial \bar{x}} \cdot \frac{\partial V(\bar{x}-n \rho-\tau)}{\partial \bar{x}}\right\} d \bar{x}
\end{array}
$$

Taking the Fourier transform of (12), and writing the summation as an average, yields the replay signal power spectral density

$$
P(k)=\frac{1}{\rho}\left\{\overline{|V(k)|^{2}} \cdot\left[1+k^{2} \overline{s^{2}}\right]\right\}
$$

where $V(k)$ is given by (6) and the overbar indicates spatial averages.

Variations in the bit sizes include variations in the thicknesses of magnetic bits. These variations cause fluctuations in the head-to-medium spacing. To account for this variation, the lower limit of integration with respect to $y$ in (4) is changed to the random variable $d_{r}$ which has a mean value of $\bar{d}$, while the upper integration limit is maintained at $d+\delta$ (i.e., changes in $\delta$ are counteracted by changes in $d$ in the opposite direction, thus keeping the sum $d+\delta$ fixed). Therefore, (13) becomes

$$
\begin{aligned}
& P(k)=\frac{1}{\rho}\left\{\overline{\alpha^{2}} v^{2} \mu_{o}^{2} M^{2}\left|H_{s}(k)\right|^{2} \cdot \overline{w^{2}} \cdot \overline{\left[\frac{\sin ^{2}\left(\frac{k l}{2}\right)}{\left(\frac{k}{2}\right)^{2}}\right]}\right. \\
& \left.\cdot\left[\overline{e^{-2 k d_{r}}}-2 \overline{e^{-k d_{r}}} e^{-k(\bar{d}+\bar{\delta})}+e^{-2 k(\bar{d}+\bar{\delta})}\right] \cdot\left[1+k^{2} \overline{s^{2}}\right]\right\} .
\end{aligned}
$$

In (14), $\overline{\alpha^{2}}=1$ and it was assumed that the magnitude of the magnetization $M$ of individual islands is not changing.

To evaluate the averages in (14), a distribution of bit sizes from a patterned medium sample is required. Fig. 2 shows a SEM image of the PtCo patterned medium used in this analysis, which was produced using laser interference lithography [6], [7]. The magnetic islands produced have an average circular diameter of $190 \mathrm{~nm}$, thickness $35 \mathrm{~nm}$, and bit spacing $\rho$ of 590 $\mathrm{nm}$ yielding an areal density of $1.9 \mathrm{~GB} / \mathrm{in}^{2}$.

Using the SEM image, a distribution of bit diameters was obtained as shown in Fig. 2. For comparison, the Gaussian distribution, calculated from

$$
g(r)=\frac{1}{\sigma \sqrt{2 \pi}} e^{-(r-m)^{2} /\left(2 \sigma^{2}\right)}
$$

using the measured average bit diameter, $m$ and standard deviation $\sigma$, is also plotted in Fig. 2. It can be seen that the Gaussian distribution fits reasonably well with the measured distribution. Using the measured distribution of bit diameters as a guide, the Gaussian function will be assumed to describe the variations in bit sizes (length, width, and thickness) in the following analysis.

Evaluation of the averages in (14) according to

$$
\overline{f(r)}=\int_{r=-\infty}^{\infty} f(r) g(r) d r
$$




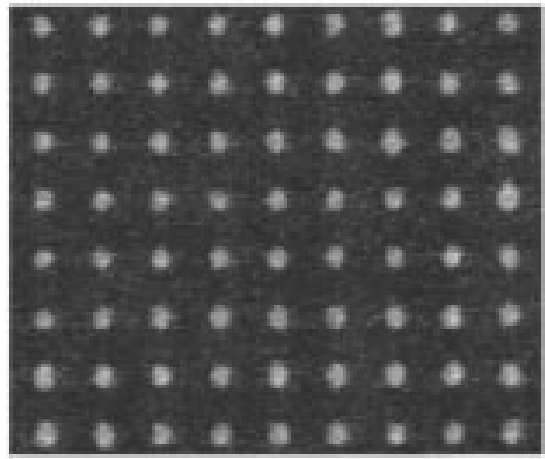

(a)

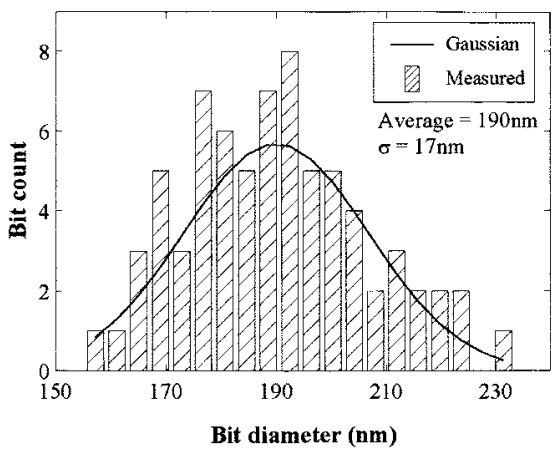

(b)

Fig. 2. SEM image of a patterned magnetic sample and measured distribution of bit diameters. Average bit diameter (length) $=190 \mathrm{~nm}$ with standard deviation $17 \mathrm{~nm}$ and bit spacing (center-to-center of $590 \mathrm{~nm}$ ).

using the distribution in (15) yields the replay signal power spectral density, including noise, as

$$
\begin{aligned}
P(k)=\frac{1}{\rho} & \left\{\frac{2 v^{2} \mu_{o}^{2} M^{2}}{k^{2}}\left|H_{s}(k)\right|^{2} \cdot\left(\sigma_{w}^{2}+\bar{w}^{2}\right)\right. \\
& \cdot\left[1-\cos (k \bar{l}) e^{-k^{2} \sigma_{l}^{2} / 2}\right] \cdot\left[1+k^{2} \sigma_{s}^{2}\right] \cdot e^{-2 k \bar{d}} \\
& \left.\cdot\left[e^{2 k^{2} \sigma_{d}^{2}}-2 e^{k^{2} \sigma_{d}^{2} / 2} e^{-k \bar{\delta}}+e^{-2 k \bar{\delta}}\right]\right\}
\end{aligned}
$$

where $\sigma_{w}, \sigma_{l}, \sigma_{s}$, and $\sigma_{d}$ are the standard deviations of the bit width, length, position jitter, and head-to-medium spacing, respectively.

Using the bit dimensions and spacings of Fig. 2, the replay spectra including bit size variations and jitter noise are plotted, respectively, in Figs. 3 and 4 using a head-to-medium spacing of $30 \mathrm{~nm}$.

Fig. 3 illustrates the effect of jitter noise alone on the replay spectrum, with all the other noise variances equal to zero. It can be seen that jitter noise affects the short wavelength components of the replay spectrum.

Bit-size variations, on the other hand, cause the disappearance of the spectral null corresponding to the bit length and a slight increase in the magnitude of the replay spectrum at long wavelengths, as indicated in Fig. 4.

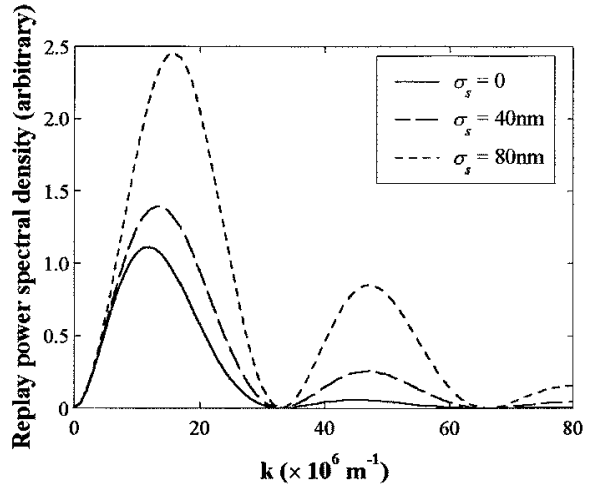

Fig. 3. Replay spectrum for increasing jitter noise variance (no bit-size variations)

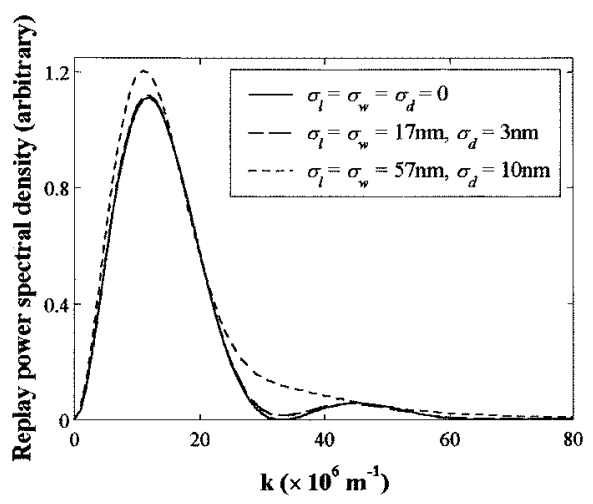

Fig. 4. Replay spectrum for increasing bit size variance (no position jitter).

\section{CONCLUSION}

General expressions for the replay signal and noise from recorded patterned media were derived. Jitter noise was found to dominate the short wavelength response of the replay spectrum. Variations in bit size and head-to-medium modulation were found to affect the long wavelength part of the replay spectrum and to result in the disappearance of the bit-length null in the spectrum. The theoretical development of signal and noise in this paper assumed inductive type replay transducers.

\section{REFERENCES}

[1] B. K. Middleton, "The recording and reproducing processes," in Magnetic Recording Technology, 2nd ed, C. D. Mee and E. D. Daniel, Eds. New York: McGraw-Hill, 1996, ch. 2, pp. 2.1-2.72.

[2] G. F. Hughes, "Read channels for patterned media," IEEE Trans. Magn., vol. 35, pp. 2310-2312, Sept. 1999.

[3] S. Shtrikman and D. R. Smith, "Analytical formulas for the unshielded magnetoresistive head," IEEE Trans. Magn., vol. 32, pp. 1987-1994, May 1996.

[4] C. D. Wright and E. W. Hill, "A reciprocity-based approach to understanding magnetic force microscopy," IEEE Trans. Magn., vol. 32, pp. 4144-4146, Sept. 1996.

[5] H. N. Bertram, Theory of Magnetic Recording. Cambridge, U.K.: Cambridge Univ. Press, 1994.

[6] J. P. Spallas, A. M. Hawryluk, and D. R. Kania, "Field emitter array mask patterning using laser interference lithography," J. Vac. Sci. Technol. B, vol. 13, pp. 1973-1978, 1995.

[7] A. Fernandez, P. J. Bedrossian, S. L. Baker, S. P. Vernon, and D. R. Kania, "Magnetic force microscopy of single-domain cobalt dots patterned using interference lithography," IEEE Trans. Magn., vol. 32, pp. 4472-4474, Sept. 1996. 